\title{
Impact of C-reactive protein testing on adherence to thrice-daily antibiotic regimens in patients with lower respiratory tract infection
}

\section{*Carl Llor ${ }^{a}$, Nuria Sierrab, Silvia Hernández , Ana Moragas ${ }^{b}, M^{b}$ arta Hernándezc, Carolina Bayona ${ }^{d}$, Marc Miravitlles ${ }^{\mathrm{e}}$}

\footnotetext{
a Family physician, Primary Care Centre Jaume I, Tarragona, Spain; University Rovira i Virgili, Spain.

${ }^{\mathrm{b}}$ Family physician, Primary Care Centre Jaume I, Tarragona, Spain

' Family Medicine Trainee, Hospital Joan XXIII of Tarragona, Spain

${ }^{d}$ Family physician, Primary Care Centre Valls Urbà, Valls, Spain

e Department of Pneumology, Institut Clinic del Tòrax (IDIBAPS), Hospital Clinic, Barcelona; CIBER de Enfermedades Respiratorias (CIBERES), Spain.
}

Received 28th September 2009; revised 30th January 2010; accepted 21st February 2010; online 12th May 2010

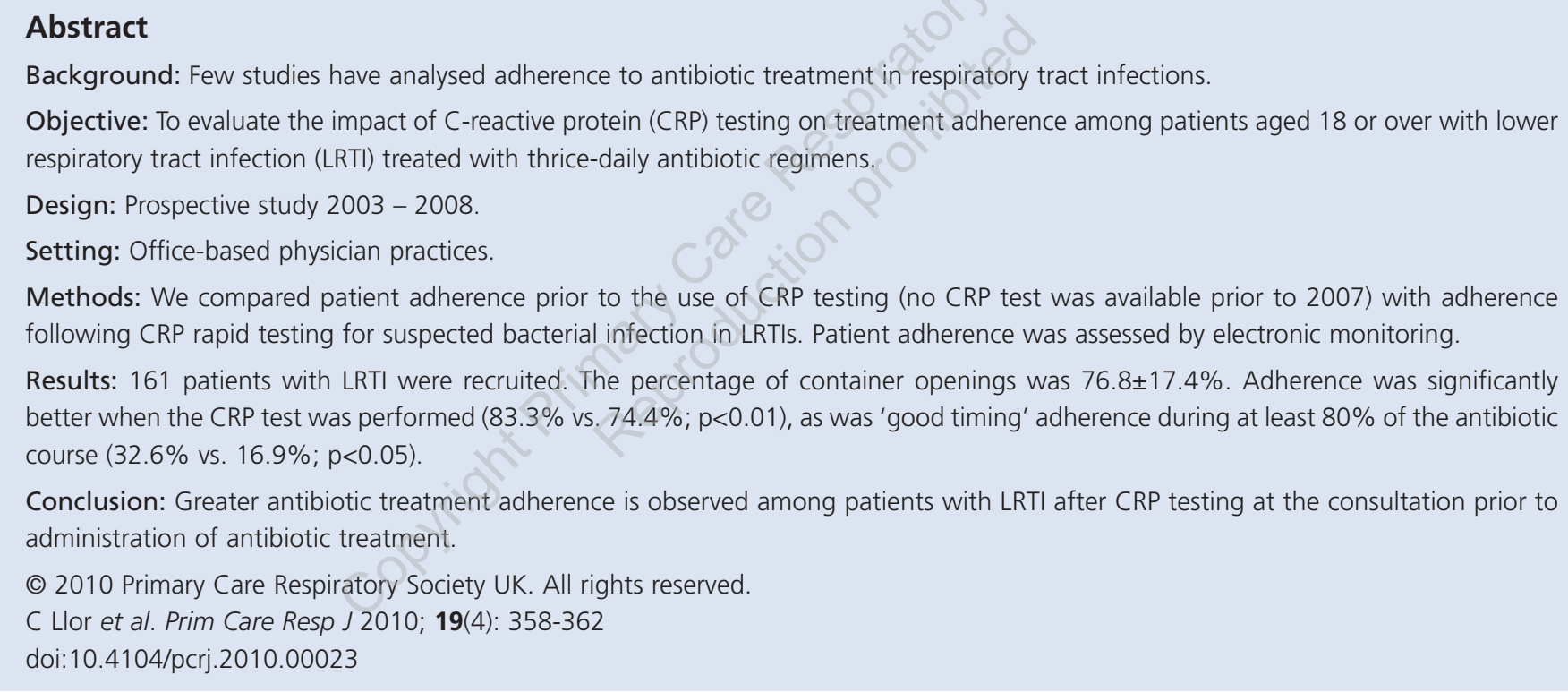

Keywords adherence, compliance, C-reactive protein, lower respiratory tract infection, antibiotics, treatment regimen

\section{Introduction}

Many studies have been performed on therapeutic adherence in chronic diseases, but there are less studies on acute illness such as infectious diseases. ${ }^{1}$ Respiratory tract infections are the most common reason for primary care consultations in industrialised countries, and antibiotics are prescribed in most cases. $^{2}$ In a meta-analysis it was found that $37.8 \%$ of patients forget to take some doses of antibiotics. ${ }^{3}$ Poor adherence with antibiotic regimens has been identified as a major cause of treatment failure. ${ }^{4}$ In addition, from a community perspective, non-adherence leads to storing of antibiotics at home, which induces self-medication, thereby favouring the emergence of bacterial resistance. ${ }^{5}$

Adherence may be measured directly and indirectly. Indirect methods evaluate adherence from the information provided by patients through measurement of events or circumstances that

\footnotetext{
* Corresponding author: $\mathrm{Dr}$ Carl Llor, c. Foixarda, 95. 43008 Tarragona, Spain
}

Tel: +34 671085857 Fax: +34 977248459 E-mail: carles.llor@urv.cat 
are probably or indirectly related to adherence. The main inconvenience of these methods is that the information is usually provided by the patients themselves, and in general they overestimate adherence. Of all the indirect methods used, overestimation of patient adherence is lowest with electronic monitoring. ${ }^{6}$

In primary care, antibiotic treatment is usually prescribed on the basis of the clinical history and physical examination, with the use of point-of-care tests being infrequent in this setting. Nevertheless, some rapid tests have been introduced in recent years to aid the clinician in making prescribing decisions. Creactive protein (CRP) testing can be used to exclude viral aetiology in lower respiratory tract infections (LRTI), and thus can lead to a reduction in the prescription of antibiotics. ${ }^{7}$ CRP rapid testing has also increased the professional satisfaction of those carrying out the tests and the patients who receive treatment. ${ }^{8}$ However, it is not known whether performing CRP rapid testing is associated with greater adherence or not. Therefore, the present study was undertaken to analyse the impact of CRP rapid testing on adherence to thrice-daily antibiotic treatment in patients with LRTI in primary care.

\section{Methods}

We performed a prospective, observational study undertaken in five general medicine outpatient clinics from 2003 to 2008. We recruited patients aged 18 years or older with suspected bacterial LRTI. Patients with uncomplicated acute illness ( $<7$ days) presenting in primary care practice with cough as the main symptom and at least two signs or symptoms of LRTI - an increase in sputum volume, sputum purulence, chest pain and/or worsening of dyspnoea - were invited to participate. In 2007 the CRP rapid test was introduced in three offices to aid the clinician in excluding viral aetiology in LRTI. All the physicians were recommended not to prescribe antibiotics with CRP levels $<20$ $\mathrm{mg} / \mathrm{L}$. However, they were free to choose whether or not to use CRP rapid testing in their consultation, whether or not to use antibiotics, and (if antibiotics were to be prescribed) were free to choose which antibiotic was to be administered.

\section{Electronic monitoring}

Two different antibiotic regimens were included in MEMS6 containers (Medication Event Monitoring System, Aardex Ltd, Zug, Switzerland) (see Figure 1). The MEMS consists of a standard container for tablets with a screw top inside which an electronic microcircuit is placed. This device records when the medication container is opened. The patients gave informed consent to participate in a study on the rational use of antibiotics, and the importance of taking all the pills in the container as per the instructions they had received was emphasised. All the patients were provided with complete information about the characteristics of the study and their participation, but were not informed at this time about the

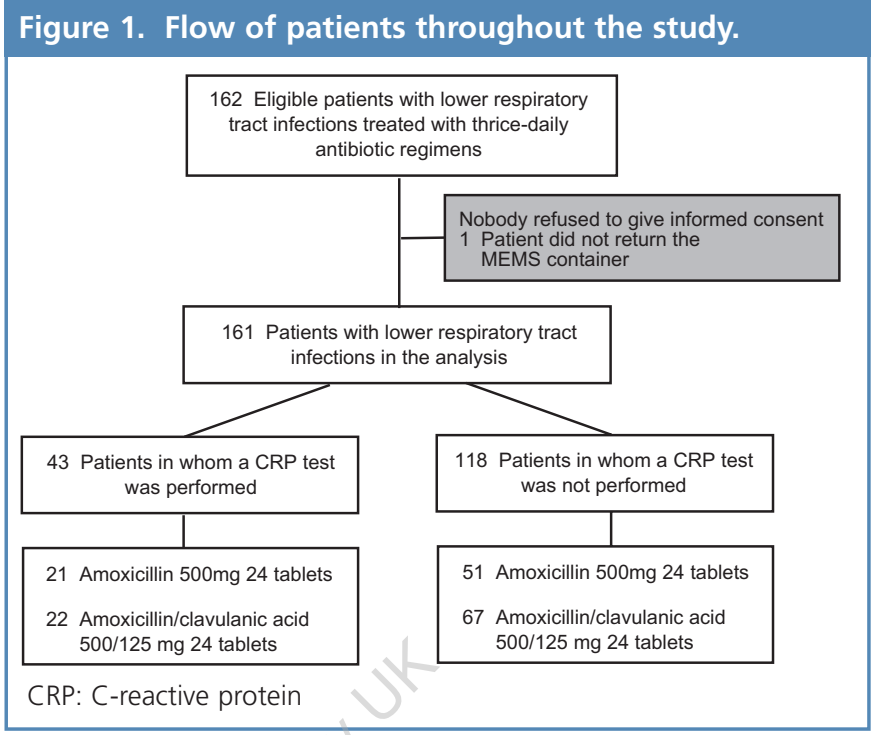

future assessment of adherence to avoid bias in the results. When they returned to the clinic, adherence was evaluated, the patients were fully informed about the results, and permission was requested to include these data anonymously in the current study. The data contained in the microprocessors were transferred to computer and processed with the PowerView program v. 1.3.2. (Aardex Ltd). Multiple openings of the container within a period of less than 15 minutes were not counted.

\section{Adherence parameters}

Three outcome measures were taken into account;

- taking adherence, calculated as the percentage of times the container was opened during the course of the treatment and the percentage of patients who opened the container at least $80 \%$ of the times, based on the pills contained in the containers,

- correct dosing, calculated as the number of days on which the patient opened the container three times daily, and

- good timing adherence during at least $80 \%$ of the antibiotic course, indicating whether the openings of the container coincided with intervals of $8 \pm 4 \mathrm{~h}$ during at least $80 \%$ of the course of antibiotic treatment.

The rate of failures was also taken into account when the patient was seen at the end of the treatment.

\section{Statistical analyses}

The study variables were analysed descriptively before applying the non-parametric Mann-Whitney tests to compare means, and the Fisher's exact test to compare categorical variables, with differences being considered as statistically significant if $p<0.05$.

\section{Results}

\section{Patient characteristics}

A total of 162 patients with LRTI were recruited. Nobody refused to give informed consent, but one patient did not 
return the container. The mean age of patients was $57.7 \pm$ 19.1 years, with 84 being females (52.2\%). Of the 161 patients with LRTI who received antibiotic therapy, CRP rapid testing was only performed prior to treatment in 43 cases (26.7\%).

Ninety-six of the 161 patients had acute exacerbations of bronchitis/chronic obstructive pulmonary disease (21 in the CRP rapid testing group and 75 in the 'no CRP' group), 61 patients had acute bronchitis (20 and 41, respectively) and 4 patients had pneumonia (2 in each group). No statistically significant differences were observed in the distribution of diagnoses between the different groups.

Figure 1 shows the flow of patients with LRTI and the antibiotic schedules received. No statistically significant differences were found in age and gender between the patients who underwent CRP testing and those who did not. Five antibiotic treatment failures (3.1\%) were observed, requiring a change in antimicrobial treatment.

Four cases demonstrated manifest non-adherence with five or less openings of the container, all due to digestive intolerance associated with amoxicillin/clavulanic acid. Eight patients opened the container more than once at intervals of $<$ 15 minutes.

\section{Data on adherence}

Table 1 shows the adherence data for patients depending on whether or not they had CRP testing in the consultation. Adherence was better when patients underwent CRP rapid testing prior to administration of the antibiotic, both in terms of the percentage of container openings $(83.3 \% \pm 14.8 \%$ vs. $74.4 \% \pm 17.7 \% ; \mathrm{p}<0.01)$ and the good timing adherence during at least $80 \%$ of the antibiotic course (32.6\% vs. $16.9 \%$; $p<0.05)$. The percentage of patients who took at least $80 \%$ of the doses was slightly better when the patient underwent rapid testing (72.1\% vs. $55.1 \%$ ), although this difference was not statistically significant.

The percentage of patients who opened the container a
Figure 2. Percentage of patients who opened the MEMS containers at least three times in lower respiratory tract infections treated with thrice daily antibiotic regimens depending on the use of C-reactive protein. Not all the patients began to take the antibiotic in the morning of the first day.

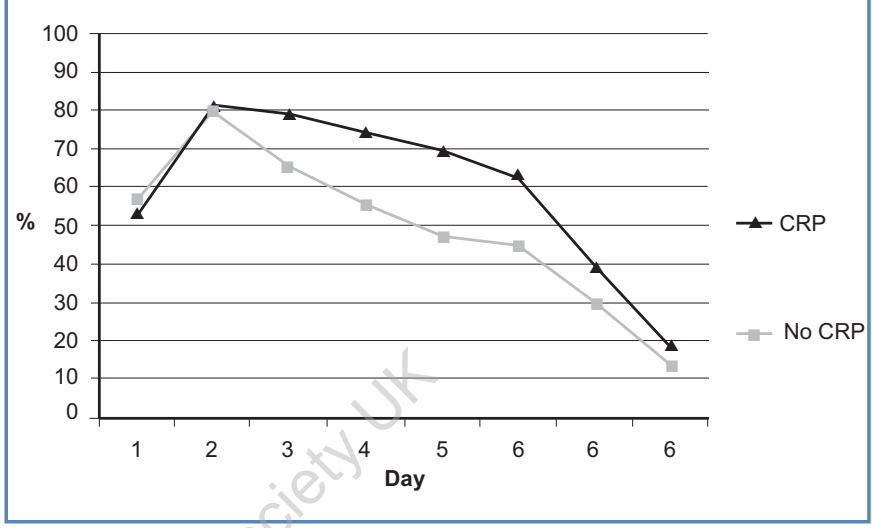

satisfactory number of times - at least three times per day throughout the treatment course - was always greater when the patient had undergone CRP testing prior to antibiotic administration (see Figure 2). The differences between those who underwent the point-of-care test and those who did not were statistically significant for days 4 and $5(p<0.01)$. A disappearance of the differences after the fifth day of the antibiotic treatment schedule was observed.

\section{Discussion}

\section{Summary of main findings}

This study demonstrates that adherence to antibiotic treatment is poor in patients with LRTI. But it also shows an association between improved adherence with antibiotic treatment and physicians' use of CRP rapid testing in the office prior to antibiotic administration (as compared to the normal practice of not using CRP testing prior to antibiotic prescribing).

Table 1. Number of container openings and adherence observed in lower respiratory tract infections treated with thrice-daily antibiotic regimens depending on the use of C-reactive protein test.

\begin{tabular}{|c|c|c|c|}
\hline Parameter & $\begin{array}{c}\text { Patients in whom a } \\
\text { CRP test was not performed }\end{array}$ & $\begin{array}{l}\text { Patients in whom a } \\
\text { CRP test was performed }\end{array}$ & All the patients \\
\hline$n$ & 118 & 43 & 161 \\
\hline Age, years. Mean (SD) & $59.5(17.6)$ & $52.9(22.1)$ & $57.7(19.1)$ \\
\hline Male gender, $\mathrm{n}(\%)$ & $55(46.6)$ & $22(51.2)$ & $77(47.8)$ \\
\hline$\%$ openings (SD) & $74.4(17.7)$ & $83.3(14.8)$ & $76.8(17.4)^{\star \star}$ \\
\hline$>80 \%$ doses, $\mathrm{n}(\%)$ & $65(55.1)$ & $31(72.1)$ & $96(59.6)$ \\
\hline Good timing adherencet $\mathrm{n}(\%)$ & $20(16.9)$ & $14(32.6)$ & $34(21.1)^{*}$ \\
\hline
\end{tabular}




\section{Strengths and limitations of this study}

This study was a prospective observational study, not a clinical trial. The objective of the study was limited to observing whether or not patients took their antibiotic treatment and determining a possible association with the use of CRP rapid testing; the objective was not specifically to compare whether performing a rapid CRP test during the consultation improved patients' adherence to antibiotic treatment.

A limitation of this study is that we cannot ensure that the disease severity of the patients included in the two groups was the same, although no significant differences were observed in the diagnoses between the two groups.

One aspect of this study which distinguishes it from other studies on antibiotic treatment adherence is that in our study we did not explain the real objective of the study until the patient returned the container and the patient was then asked to provide informed consent to use their data on adherence. The reality of including a patient in a clinical trial and explaining that treatment adherence will be monitored makes the patients feel more controlled, which can modify their behaviour. It should be pointed out that all included patients consented to the use of their data on adherence.

Another limitation is that we cannot ensure that the emphasis (on the importance of taking the antibiotic treatment) given by the physician to the patient was the same between those patients undergoing CRP testing and those not undergoing the test. However, the need to take all the pills in the MEMS container every eight hours was emphasised in all cases, as was the importance of taking all the treatment even if the patient felt well. Yet the emphasis given may have differed in the two groups despite the information being the same. Butler et al reported that many physicians were enthusiastic towards the use of these rapid tests since they could reinforce the advice that prescribing an antibiotic in negative cases was unnecessary, thereby helping convince the patient not to take treatment. ${ }^{9}$ It is also possible that when the result was positive with a high CRP concentration, the physician was more convinced of the need for the patient to take the medication.

Neither was the objective of this study to evaluate the effectiveness of the treatments administered, although five cases of treatment failure were reported which required a change in antibiotic treatment. The diagnosis in most of the cases was clinical and, although clinical presentations with a supposedly bacterial aetiology were included, it cannot be guaranteed that all the infections included were actually of bacterial origin. The physicians in this study were free to use the antibiotic that they considered to be the most appropriate.

Furthermore, it cannot be guaranteed that each time the MEMS container was opened the medication was actually taken. Nonetheless, we believe that the electronic method used and the fact that the patients were not informed as to the real objective of the study until the second visit (a method which has not been used previously) undoubtedly constitutes the greatest strength of this study. This may explain why our data on adherence are worse than those of other studies published on respiratory tract infection in which electronic monitoring was also used. . $^{10-12}$

To our knowledge this is the first time that an association between the use of CRP rapid testing and better adherence outcomes has been described. Since this is not a formal clinical trial no causal relationship may be inferred, but we have demonstrated an association between the use of this rapid test and adherence.

The ability to explain to patients that they have a greater probability of presenting a bacterial infection if their CRP test is high may be clearly persuasive regarding the need to take the medication. However, it was curious to observe that the greater adherence observed with the use of rapid testing was only observed on the first days of antibiotic treatment and was no longer significant after the fifth day. It is likely that after a few days of feeling well the patients discontinue taking the antibiotic treatment regardless of whether they had undergone the rapid testing.

\section{Implications for further research}

In most clinical situations, particularly in the case of antibiotic therapy, the time interval between doses is extremely important. In most cases, the pharmacokinetics of the drugs makes it necessary for them to be taken not later than indicated to ensure maximum therapeutic action. ${ }^{13} \mathrm{~A}$ prolonged time interval between doses has been found to lead not only to reduced effectiveness, but also to the emergence of antibiotic-resistant pathogens. ${ }^{14}$ The factors which most influence patients to take their medications appropriately are the number of daily doses and the duration of the treatment. In a previous study with fewer cases we have reported that non-adherence is greater the higher the number of antibiotic doses per day in LRTIs. ${ }^{15}$ Despite not being a formal clinical trial, the present study demonstrates how a simple technique such as the performing of CRP testing prior to the prescription of an antibiotic is associated with greater therapeutic adherence. The results of this study should be of interest to researchers designing future clinical trials to determine whether a strategy such as that reported here is able to improve therapeutic adherence to antibiotic treatment.

\section{Funding body}

The MEMS containers were provided through a grant from GlaxoSmithKline. The CRP testing was provided free of charge by Axis Shield and Orion Diagnostica. The authors declare that they did not receive any direct economic support for undertaking this study. 


\section{Competing interests}

None declared.

\section{References}

1. McDonald HP, Garg AX, Haynes RB. Interventions to enhance patient adherence to medication prescriptions. Scientific review. JAMA 2002;288(22): 2868-79. http://dx.doi.org/10.1001/jama.288.22.2868

2. McCraig LF, Hughes JM. Trends in antimicrobial drug prescribing among officebased physicians in United States. JAMA 1995;273(3):214-19.

3. Kardas P, Devine S, Golembesky A, Roberts C. A systematic review and metaanalysis of misuse of antibiotic therapies in the community. Int J Antimicrob Agents 2005;26(2):106-13. http://dx.doi.org/10.1016/j.ijantimicag.2005.0 4.017

4. Sclar DA, Tartaglione TA, Fine MJ. Overview of issues related to medical adherence with implications for the outpatient management of infectious diseases. Infect Agents Dis 1994;3(5):266-73.

5. Vrijens B, Urquhart J. Patient adherence to prescribed antimicrobial drug dosing regimens. J Antimicrob Chemother 2005;55(5):616-27. doi:10.1093/jac/dki066

6. Osterberg L, Blaschke T. Adherence to medication. N Engl J Med 2005;353(5); 487-97. http://dx.doi.org/10.1056/NEJMra050100

7. Cals JW, Butler CC, Hopstaken RM, Hood K, Dinant GJ. Effect of point of care testing for $C$ reactive protein and training in communication skills on antibiotic use in lower respiratory tract infections: cluster randomised trial. BMJ 2009;338:b1374. http://dx.doi.org/101136/bmj.b1374.

8. Kolmos HJ, Little P. Controversies in management: should general practitioners perform diagnostic tests on patients before prescribing antibiotics? BMJ
1999;318(7186):799-802.

9. Butler CC, Simpson S, Wood F. General practitioners' perceptions of introducing near-patient testing for common infections into routine primary care: a qualitative study. Scand J Prim Health Care 2008;26(1):17-21. http://dx.doi.org/10.1080/02813430701726285

10. Cals JW, Hopstaken RM, Le Doux PH, Driessen GA, Nelemans PJ, Dinant GJ. Dose timing and patient adherence with two antibiotic treatment regimens for lower respiratory tract infections in primary care. Int J Antimicrob Agents 2008; 31(6):531-6. http://dx.doi.org/10.1016/j.ijantimicag.2008.01.029

11. Favre O, Delacrétaz E, Badan M, Glauser M, Waeber B. Relationship between the prescriber's instructions and adherence with antibiotherapy in outpatients treated for an acute infectious disease. J Clin Pharmacol 1997;37(3):175-8.

12. Kardas P. Comparison of patient adherence with once-daily and twice-daily antibiotic regimens in respiratory tract infections: results of a randomized trial. J Antimicrob Chemother 2007;59(3):531-6. http://dx.doi.org/10.1093/jac/ dkl528

13. Craig WA. Pharmacokinetic/pharmacodynamic parameters: rationale for antibacterial dosing of mice and men. Clin Infect Dis 1998;26(1):1-12. http://dx.doi.org/10.1086/516284

14. Thomas JK, Forrest A, Bhavnani SM, et al. Pharmacodynamic evaluation of factors associated with the development of bacterial resistance in acutely ill patients during therapy. Antimicr Agents Chemother 1998;42(3):521-7.

15. Llor C, Sierra N, Hernández S, et al. The higher the number of daily doses of antibiotic treatment in lower respiratory tract infection the worse the adherence. J Antimicrob Chemother 2009;63(2):396-99.

\section{Available online at http://www.thepcrj.org}

